\title{
Real-time optical measurements with picosecond resolution during laser induced transformations
}

\author{
J. Solis, ${ }^{\text {a) }}$ J. Siegel, and C. N. Afonso \\ Instituto de Optica, CSIC, Serrano 121, 28006-Madrid, Spain
}

(Received 10 August 1999; accepted for publication 21 December 1999)

\begin{abstract}
This work describes a novel technique allowing to measure with picosecond resolution the time evolution of the optical properties (reflectivity/transmission) of a material surface exposed to a single laser pulse. The experimental setup is based on the use of a streak camera in combination with a single-mode probe $\mathrm{cw}$ laser. The maximum achievable time resolution is about $1 \mathrm{ps}$ for the acquisition of a single event. Results concerning melting of Ge films under ps pulses will be used to illustrate the potential of this measurement technique for resolving single optical transient events in the ps time scale. The advantages of the system with respect to the use of pump-and-probe optical measurements are especially remarkable for the study of events occurring in time windows with full widths from 1 to $10 \mathrm{~ns}$, as it is the case for rapid solidification phenomena induced by ultrashort laser pulses. This has allowed us to investigate in detail, for instance, the occurrence of bulk and surface initiated solidification phenomena in thin amorphous Ge films. (C) 2000 American Institute of Physics. [S0034-6748(00)03304-9]
\end{abstract}

\section{INTRODUCTION}

Optical measurements with time resolution in the ps/fs time scales have revolutionized our comprehension of lasermatter interaction ${ }^{1}$ and of the physics of semiconductors and nanoscale structured materials, ${ }^{2}$ among other fields in physics, chemistry, or biology. ${ }^{3}$ The most common approach to achieve such time resolution is the use of the so-called pump-and-probe scheme in which a short (ps/fs) pump pulse repetitively excites the material while a probe pulse, in the simplest configuration, a lower intensity pulse split from the рump and temporarily delayed with respect to it, is used to measure the instantaneous optical response of the excited surface.

The term optical response refers in general to a wide variety of parameters which can be explored by different modifications of this simple scheme and which may include, among others, the analysis of the induced changes in surface reflectivity, transmission, absorption, Raman scattering, fluorescence, or in the intensity of a conjugated beam in the case of a four-wave mixing experiment. Most of these pump-andprobe spectroscopic techniques are described in Ref. 2 and those cited therein. It is important to notice though, that for a single interaction event, a pump-and-probe experiment provides a single data point of the time reconstruction of the signal and thus the process must be repeated several times, scanning the time delay between the pump and the probe pulses in order to perform the full time reconstruction of the signal. This is also necessary, for a given delay value, in order to average-out noise effects and to discard those measurements associated to pump pulses whose fluence is out from a maximum acceptable statistical deviation. The ultimate time resolution of the experiment is normally given by

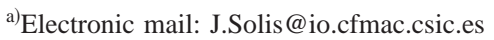

the duration of the probe pulse itself but not always, as in the case of up-conversion luminescence spectroscopy. ${ }^{2}$

In most cases, for instance, the study of excitation dynamics in solids, the use of pump-and-probe measurements turns out to be a relatively simple and powerful technique for the time reconstruction of an optical transient event. However, when the excitation pulse fluence used is high enough to induce permanent changes in the irradiated surface, as it happens in laser annealing, ${ }^{1,4}$ ablation, ${ }^{5,6}$ and optical recording ${ }^{7-9}$ the use of pump-and-probe measurements becomes much more difficult since the time reconstruction requires the exposure of a "fresh region" of the surface for each pair of pump and probe laser pulses. ${ }^{10}$ This makes it necessary the use of samples with good structural, compositional, and optical homogeneity over relatively large areas, which in some cases can be a serious drawback. ${ }^{11}$ Furthermore, the information obtained over a full delay range only corresponds to a single data point of the fluence spectrum except in very particular experimental arrangements, like ps/fs time-resolved optical microscopy. ${ }^{12}$ The need of a large number of irradiations over fresh regions of the sample is particularly necessary in situations in which the dynamics of the transformation to be analyzed depends critically on the laser pulse fluence, ${ }^{11,13,14}$ making also the requirements for the stability of the laser pulse source to be very demanding. Finally, most of the essential information concerning rapid solidification phenomena under ultrashort laser pulses is contained in a time window with a full width typically between 1 and $10 \mathrm{~ns} .{ }^{15}$ This time window corresponds to optical delays in the $0.30-3.0 \mathrm{~m}$ range. The scan of a delay line over such a distance with a sufficient number of data points in a pump-and-probe experiment is, in addition to the problems above mentioned, normally complicated and also tedious. Furthermore, even in the case of using fast photodetectors 
and digitizers, this time window is hardly accessible in a single event experiment.

The use of streak cameras ${ }^{16,17}$ allows to combine in principle both single exposure acquisition and high (even sub-ps) time resolution. However, to our knowledge, there is only one work describing a single exposure reflectivity measurement using a streak camera. ${ }^{18}$ The authors used as the probe beam an ultrahigh repetition $(\approx 10 \mathrm{GHz})$ train of 30 ps pulses obtained by passing a portion of the pump pulse through an air-gap etalon. The time resolution of the experiment was thus not limited by the time resolution of the streak camera but by duration of the probe pulses ( $30 \mathrm{ps}$ ), while the time separation between consecutive data points was even higher (55-135 ps). In addition, because of the way the probe pulses train was generated, it was of nonconstant amplitude, forcing to perform a very careful normalization of the raw reflectivity data to the actual amplitude envelope of the pulse train.

In this work, we report the first single exposure optical measurements with ps resolution during laser induced phase changes obtained by means of a streak camera and using a true $\mathrm{cw}$ probe laser. Besides to the most important advantage already mentioned with respect to repetitive pump and probe experiments (i.e., single exposure acquisition), the proposed system has not any wavelength constrain for the probe light beam since the pump and the probe laser beams are completely independent. The same applies for the duration of the probe pulse within certain limits which will be later discussed. The time resolution is only limited by that of the streak camera used, provided that some critical experimental requirements are fulfilled. As a consequence, its use for the analysis of structural transformation dynamics in the ps/ns time scales opens important perspectives for the comprehension of nonequilibrium phase transitions.

\section{EXPERIMENT}

The scheme of the experimental setup used can be seen in Fig. 1. It essentially consists of a pump laser system used to irradiate the sample surface in a single pulse exposure, a $\mathrm{cw}$ probe laser used to monitor the time evolution of the sample reflectivity (or transmission) upon irradiation, and a light detection system with time resolution. Different optical and electronic elements are used to control the temporal and spatial profile, the fluence, and the synchronization of the pump and the probe lasers beams. In the following, we will concentrate on the description of its most important elements.

\section{A. The light detection system}

The reflectivity measurements with ps resolution have been carried out by means of a Hamamatsu Universal Streak Camera (Model C5680 equipped with single sweep unit (Model M5676)). The input optics transmisive window is $200-1600 \mathrm{~nm}$, while the streak tube sensitive window is $200-850 \mathrm{~nm}$. The nominal time resolution of the system is $1.44 \mathrm{ps}$ in a time window of $200 \mathrm{ps}$. Since the longest time window of this particular sweep unit is $50 \mathrm{~ns}$, we have alternatively used a fast amplified photodiode in combination

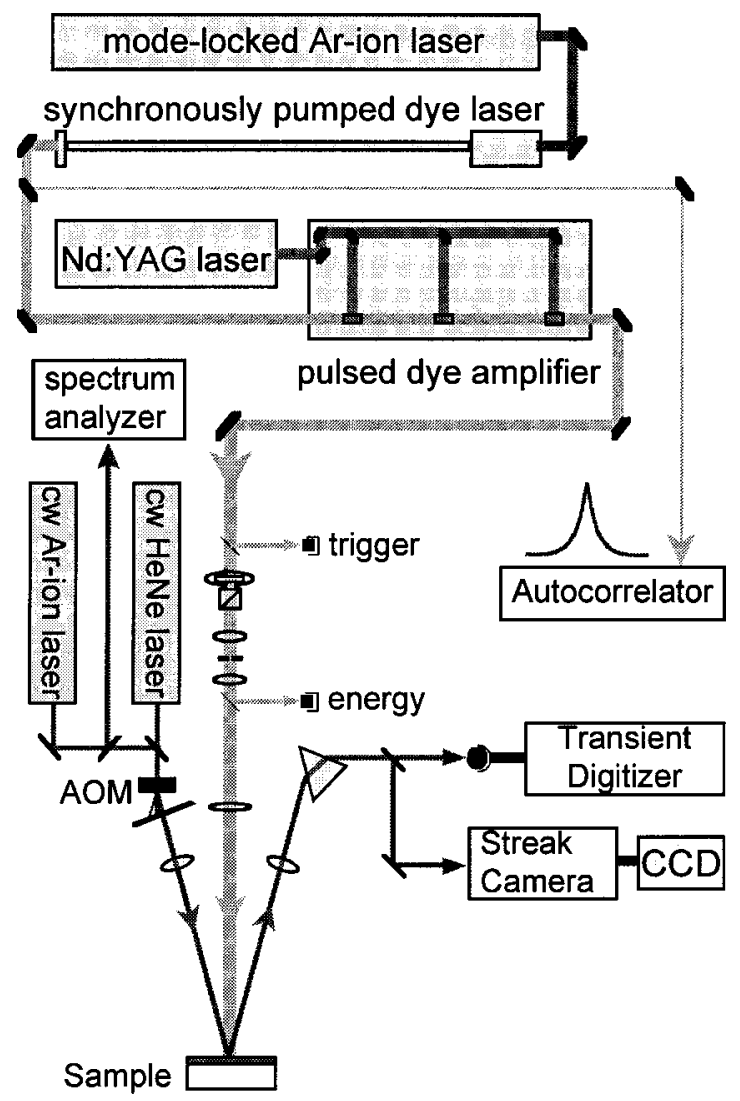

FIG. 1. Experimental setup for real-time reflectivity/transmission measurements upon ps laser pulse induced structural transformations in materials. The configuration shown corresponds to reflectivity measurements.

with an oscilloscope or a transient digitizer ${ }^{19}$ in order perform measurements over longer time windows and also to analyze the influence of the time resolution in obtained results. The time resolution of this later system is a few ns.

It is out of the scope of this work to describe in detail the operation of a streak camera. Thus, we will limit ourselves to describe its basic principles of operation emphasizing those aspects which affect the actual time and intensity resolution achievable in our particular application and that are discussed in detail in Sec. II E.

The light signal to be measured is focused to a small spot size at the entrance slit of the streak camera. The light spot at this horizontal slit is then imaged one by one on the surface of a photocathode. The photoelectrons there generated, whose number is proportional to the incident light instantaneous intensity, are then extracted, accelerated, and swept vertically by a fast vertical voltage ramp. This way the time dependence of the intensity of the input light beam is converted in a spatial electron distribution along the vertical axis. The electrons reach then a microchannel plate (MCP) were the electron spatial distribution is amplified and transferred to a phosphor screen. The latter converts the electron signal in a light intensity distribution which is imaged by a cooled charge coupled device (CCD) camera, allowing to perform a quantitative analysis of the time evolution of the signal.

There is obviously a minimum time separation between two different optical events exciting the same spot of the 
photocathode below which their corresponding electron distributions spatially overlap on the microchannel plate. This minimum time separation (resolution) is dependent in itself on the sweep speed, and on the spot size of the light beam input at the entrance slit of the device. The standard way to determine the time resolution of a streak camera is to determine the apparent pulse width of an instantaneous event (a very short pulse, $100 \mathrm{fs}$ or less) for the different sweep speeds (time windows). In our case, for the shortest time window of the system (200 ps), the time resolution determined is $1.44 \mathrm{ps}$, while for the longest one $(50 \mathrm{~ns})$, the time resolution is about $350 \mathrm{ps}$. These nominal resolutions are achieved when the input beam spatial width is $\leqslant 30 \mu \mathrm{m}$ at the entrance slit of the system.

When the streak camera is operated in single sweep mode (or low repetition mode) and a cw or nearly cw beam is used, gating of the photocathode is required to reduce the internal photoelectron scattering in the streak tube. The excess amount of nonswept photoelectrons at the presweep waiting position may cause an overflow onto the early portion of the sweep, affecting detrimentally the time resolution of the system. These space charge effects are also observed if the power of the input beam is too high, making the time resolution also to be dependent on the input light intensity. This effect makes necessary to define a parameter denominated dynamic range of the system as the ratio of the intensities of the minimum detectable signal to the one generating an apparent temporal broadening of $20 \%$ in the signal. Actually, the upper limit on the streak camera's dynamic range is mostly limited by the space charge effect at the sweep deflection plates. ${ }^{20-22}$ The dynamic range is also a function of the time window of the system and ranges typically from 1:50 to $1: 1000$ for time windows of $200 \mathrm{ps}$ to $20 \mathrm{~ns}$, corresponding to resolutions of $1.4-140 \mathrm{ps}$, respectively.

\section{B. The irradiation (pump) laser system}

The core of the pump laser system is formed by an actively mode-locked $\mathrm{Ar}^{+}$ion laser which synchronously pumps a dye (Rhodamine $6 \mathrm{G}$ ) oscillator. The latter provides tunable laser pulses with a typical duration of $10 \mathrm{ps}$ at a repetition rate of $82 \mathrm{MHz}$ and with an energy per pulse in the $n J$ range. This pulse train is fed in a pulsed dye (sulforhodamine B) amplifier pumped by a low repetition rate frequency-doubled Nd-YAG laser. The output of this system is a high energy laser pulse $(\approx 350 \mu \mathrm{J})$ with a repetition rate variable from single shot up to $10 \mathrm{~Hz}$. The amplified pulse duration is 30 ps while the amplified spontaneous emission to amplified laser signal ratio is typically below $3 \times 10^{-3}$. Great care is always taken in order to achieve optimum synchronous pumping conditions of the dye laser oscillator ${ }^{23}$ and a proper synchronization of the seed pulses to the Nd-YAG laser pulses which was achieved by monitoring the laser pulses of the oscillator and the amplifier by means of an autocorrelator and the streak camera, respectively. The amplified pulses travel through different optical elements for energy regulation and spatial filtering before being focused at the sample surface to a size of a several hundreds of microns, leading to fluences ranging typically from $10 \mathrm{~mJ} / \mathrm{cm}^{2}$ to $1 \mathrm{~J} / \mathrm{cm}^{2}$. The refined procedure to determine the fluence at the sample site has been described in detail elsewhere. ${ }^{24,25}$ It is remarkable that the reflectivity measurements and their time resolution are completely independent of the pulsed laser source generating the pump pulse.

\section{The probe laser system}

The probe laser used in combination with the streak camera for the real-time optical measurements with ps resolution is a single-mode $\mathrm{cw} \mathrm{Ar}^{+}$laser. As mentioned above, in order to analyze the influence of time resolution in the results and also for analyzing time windows well above 100 ns, we have also used a fast amplified photodiode in combination with a transient digitizer. In that case, the probe laser used was either the former or a multimode HeNe laser. In both cases, the probe laser output traverses an acousto-optic modulator in order to generate a square light pulse with a duration of typically $1 \mu \mathrm{s}$ and with a peak power that can range up to maximum values of $3 \mathrm{~mW}$ (HeNe laser) or 100 $\mathrm{mW}$ ( $\mathrm{Ar}^{+}$laser). This light pulse is used to monitor the reflectivity or transmission evolution at the center of the irradiated region using a prism in order eliminate by chromatic dispersion any possible spurious contribution to the reflectivity/transmission signal caused scattering of the pump beam. The large monochromaticity of the probe pulse as well as the short glass path along the prism ensures that the time evolution of reflectivity/transmission signal is not affected by the chromatic dispersion which the probe beam undergoes in the prism.

In order to monitor the evolution of a region homogeneously irradiated the probe beam is focused to a spot size about ten times smaller $(50 \mu \mathrm{m})$ than that of the pump beam at the sample surface. In spite of the relatively tight focusing, the pulse duration used is sufficiently short to avoid the $\mathrm{cw}$ annealing of the surface. The procedure used to determine the surface temperature increase caused by the probe pulse has been described elsewhere. ${ }^{26}$ Even for a badly thermal conducting material as glass, and for an absorption around $50 \%$, the maximum temperature increase induced does not exceed $10 \mathrm{~K}$, a value negligible when compared to the melting point of most materials under study. The use of a square probe pulse also provides a direct amplitude reference in order to measure quantitatively the reflectivity changes induced by the pump pulse. Finally, as we will see later, a pulse of $1 \mu$ s is sufficiently short to avoid space charge effects in the photocathode of the streak camera which would deteriorate the ultimate time resolution achievable.

For the case of the He-Ne laser, longitudinal mode beating effects generate a characteristic quasi-periodical noise with a central frequency around $500 \mathrm{MHz}$. This generates a very noisy reflectivity signal in the ns time scale. This detrimental effect associated to the use of multimode probe beams is well known long since ${ }^{27}$ and can be partially compensated by electrical filtering of the signal using notch filters $^{28}$ or by post-processing the signal using Fourier analysis. ${ }^{19}$ In both cases, the price to be paid for eliminating frequency components of the signal is a loss in the time resolution achievable. A HeNe laser in combination with a 


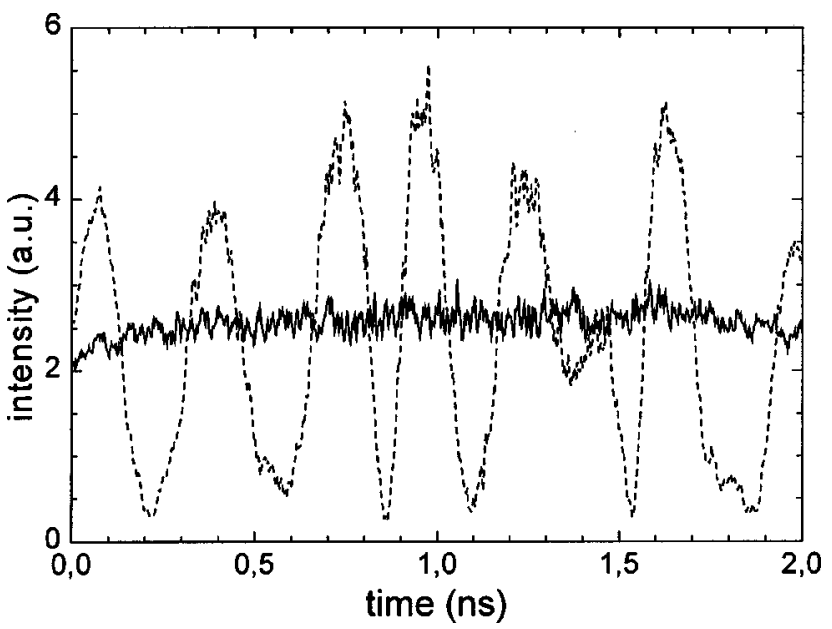

FIG. 2. Time evolution of the $\mathrm{cw} \mathrm{Ar}^{+}$probe laser intensity in single (solid line) and multimode (dashed line) operation as measured with the Streak Camera.

fast photodiode and a transient digitizer (or an oscilloscope with enough bandwidth) is thus sufficient to discriminate reflectivity changes in the order of $1 \%$ if the time resolution required is above some $5 \mathrm{~ns}$ or the time window to be analyzed is close to or longer than $100 \mathrm{~ns}$.

In the case of the single-mode $\mathrm{Ar}^{+}$ion laser, the suppression of mode competition by an intracavity ethalon allows to achieve a smooth time intensity profile for the probe pulse both in ns and ps time scales. This can be clearly seen in Fig. 2, that shows two typical streak camera traces of the time evolution of the probe laser output in single-mode and multimode operation over a time window with a full width of $2 \mathrm{~ns}$. In the latter case, mode competition gives rise to the appearance of random intensity oscillations with an enormous modulation depth making it impossible to discriminate those intensity variations associated to reflectivity/ transmission changes caused by the pump pulse. The temporal flatness of the probe pulse in the sub-ns time scale is actually one of the key factors to achieve accurate reflectivity measurements in a single exposure with the desired time resolution. In principle, any very stable single-mode laser at the desired wavelength could be used as probe beam, provided that the output power is sufficient. The apparent curvature of the traces shown in Fig. 2 at the extremes of the time window is due to the so-called "shading effects" in the streak camera which will be discussed in Sec. IIE.

\section{Synchronization}

The pump-and-probe pulses are synchronized in such a way that the later reaches the surface of the sample about $300 \mathrm{~ns}$ before the arrival of the pump. This is done by using a signal synchronous to the firing of the flash lamps of the Nd-YAG laser to drive a delay generator which controls the acousto-optic modulator. This trigger signal is sufficiently advanced $(\approx 200 \mu \mathrm{s})$ with respect to the amplified pulse output and shows a jitter of a few tens of ns with respect to it, completely negligible when compared to the duration of the probe pulse $(\approx 1 \mu \mathrm{s})$.
The synchronization of the streak camera trigger with respect to the pump-and-probe pulses is more complicated. At this point, it is important to bear in mind that there exists a relatively large delay between the arrival of the trigger signal to the streak camera to the time the sweep voltage ramp is applied. This delay can range from $\approx 14$ to $\approx 80 \mathrm{~ns}$ depending on the selected time window. If the streak camera is optically triggered, this makes it necessary the use of an optical delay line of several meters in the pump beam path between the location where the trigger signal is detected and the sample surface. To avoid this, we have triggered the streak camera using an electrical signal provided by a synchronization module. This module uses as inputs the $82 \mathrm{MHz}$ $\mathrm{Ar}^{+}$ion laser oscillator clock frequency and the $10 \mathrm{~Hz}$ oscillator clock frequency of the Nd-YAG laser and provides an output which is synchronous with the amplified output pulse but advanced with respect to it about 100 ns. This advanced trigger signal shows a jitter below $150 \mathrm{ps}$ and can be thus used with no problem for time windows with full widths down to $500 \mathrm{ps}$. For the shortest one (200 ps), sometimes the transient signal is not within the observation window. This approach (electrical trigger) can be used in many other amplified laser systems (like regenerative amplifiers) provided that the jitter between the pretrigger signal (Pockels cell trigger, for instance) and the amplified output pulse is smaller than one half of the time window used in the streak camera.

\section{E. Factors affecting the time and intensity resolution of the experiment}

As we have mentioned in Sec. II A, the determination of the actual time resolution of a streak camera when monitoring a nearly $\mathrm{cw}$ beam is not trivial since the definition of the concept of resolution is not easy to establish in this condition. By fortune, the presence of space charge effects can be easily detected by following the evolution of the horizontal width of the trace in the streak images as a function of the probe pulse width/intensity. The width of the trace increases considerably as soon as the intensity of the probe pulse is above the threshold for the formation of a cloud of charge in the photocathode. Since space charge effects in our case are mainly caused by the light reaching the photocathode before the extraction voltage is applied, it should be in principle possible to eliminate them by gating the photocathode. We have found that the synchronization of the photocathode gate, the voltage ramp, and the pump-and-probe pulses is extremely difficult in single shot operation and we have not been able to eliminate completely the presence of space charge effects by this means. In turn, the reduction of the probe pulse duration to approximately $1 \mu$ s in combination with the use of a moderate power completely eliminates this effect and also helps to preserve the photocathode lifetime, which can be strongly reduced by long time exposure to a $\mathrm{cw}$ beam. The careful control of the intensity and duration of the probe beam pulse and thus, the elimination of space charge effects ensures that the time resolution of the experiment in a given time window corresponds to the nominal time resolution of the streak camera. 
Finally, there are two important factors affecting the light intensity resolution of the experiment (reflectivity/ transmission sensitivity). They are the optical aberrations of the streak camera globally considered as an image forming system and the different sources of noise in the reflectivity signal detected by the streak camera.

The first of them can be easily understood if we consider how the streak image of a perfectly uniform light source in space and time covering all the extension of the entrance slit of the streak camera should look like. If the streak camera were a perfect image forming system, the corresponding image should be a bidimensional intensity distribution perfectly flat. However, aperture effects in the different elements of the streak (input optics, photocathode, MCP, phosphor, CCD camera) along with small spatial inhomogeneities in their sensitivity/transmission distort this perfect image. Actually, the image becomes something very much like an elliptical disk with a flat intensity distribution in a broad central region and with an intensity decay towards the edges. The intensity distribution also shows a number of point defects in which the intensity is either higher or lower than the average of the surrounding distribution. All these effects are globally designated as "shading" and can be clearly appreciated in Fig. 2 (where they have not been corrected) by the curved shape of the trace near the extremes of the time window. This curved shape is a consequence of sensitivity decrease of the image forming system towards the edges. The way to correct the shading effects is to normalize the streak image by one obtained under illumination with a "perfectly time and space homogenous light source," what is called a shading correction image. The latter, to a good approximation, can be obtained using a white incoherent light source illuminating a fine light diffuser in front of the entrance slit of the streak camera.

This approach works very well when the streak camera is operated in synchroscan mode (i.e, high repetition and averaging) but the results are not so good in single sweep operation. There are several reasons for this. First, the shading correction image has to be obtained exactly in the same conditions (sweep speed, MCP, and CCD camera gain) in which the experiment is carried out. Second, the temporal homogeneity of an incoherent white light source is real only when it is averaged over a sufficiently long period of time or a sufficiently large number of sweeps but this is not true for a single exposure in a given time window. Third, an incoherent continuous light source generates a very small number of photons over a time window of a few hundreds of ps or ns, making it very hard to achieve exactly the same gain conditions in which the probe beam detection is carried out in the experiment. In order to correct the shading effects, we have normalized the single exposure streak images of the probe beam by an image obtained with the pump beam blocked. The results obtained using this method for single exposure images are normally much better than the ones obtained using the standard procedure, especially for correcting the sensitivity decay near the edges.

The second factor affecting the resolution of the intensity variations measured are the different optical and electrical noise sources present. In all cases, the streak images ac-

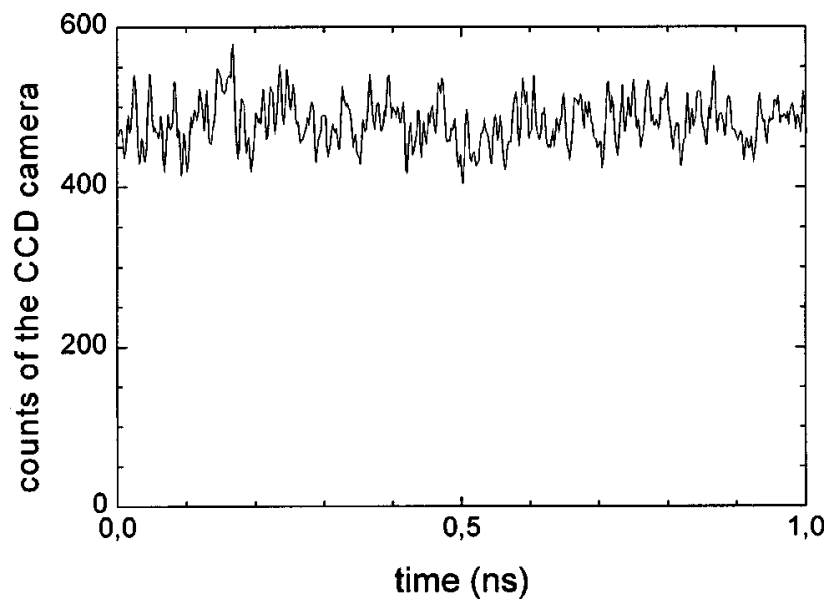

FIG. 3. Time evolution of the $\mathrm{cw} \mathrm{Ar}^{+}$probe laser intensity as seen in a time window of $1 \mathrm{~ns}$ showing the characteristic amplitude of the noise band.

quired have been background subtracted, i.e., subtracted by an image obtained in complete darkness by closing the streak camera shutter) in order to eliminate dark signal effects. Even after this correction, the signal-to-noise $(\mathrm{S} / \mathrm{N})$ ratio observed when measuring the probe beam intensity evolution ranges from 50:1 to 10:1 depending on the time window used. Therefore, the minimum resolvable reflectivity change can range from $2 \%$ to $10 \%$ with respect to the reflectivity level of the nonexcited surface. The noise observed in the reflectivity traces appears typically in the form of a more or less periodic modulation overimposed to the intensity evolution of the probe beam as can be seen in Fig. 3. The latter shows a reflectivity trace in the absence of a pump pulse in a time window of $1 \mathrm{~ns}$. The typical separation between maxima and minima in the noise band is independent on the time window used, which indicates that is not related to a modulation in the probe beam intensity. Also the $\mathrm{S} / \mathrm{N}$ ratio decreases as the time window becomes shorter or the MCP gain increases. If we consider that the dynamic range specification for the streak camera used ranges approximately from 50 to 1000 depending on the time resolution, and that the minimum amount of detectable signal corresponds to about eight electrons at the photocathode with a quantum efficiency $\approx 0.025$, it can be deduced that with a power of about $1-10 \mathrm{~mW}$ for the probe beam reaching the entrance slit of the photocathode, there is sufficient number of photons for resolving a transient change in the reflectivity signal of about $2 \%$ in a time window of 200 ps. It is thus clear that the observed $\mathrm{S} / \mathrm{N}$ ratio is not related to the amount of photons available in the probe beam in the corresponding time window, even in the shortest one. The main noise source are the photons arriving at the photocathode before the sweep ramp is applied and which give rise to random fluctuations of the streak image intensity which are overimposed to the reflectivity signal. Their effect can be reduced by smoothing the transient signal without affecting very much the time resolution of the signal. This is possible because the typical separation in pixels between noise spikes is smaller than the corresponding time resolution of the system. In any case, the $\mathrm{S} / \mathrm{N}$ ratio finally achieved is always a compromise between the probe intensity required for maximizing the signal and 


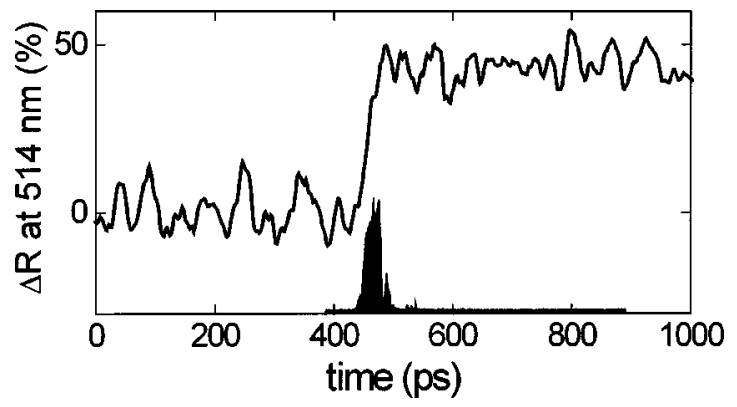

FIG. 4. Transient reflectivity increase at $514 \mathrm{~nm}$ upon melting of the $a$-Ge film by a single ps laser pulse $\left(65 \mathrm{~mJ} / \mathrm{cm}^{2}\right)$ as measured by a Streak Camera in a time window of $1 \mathrm{~ns}$. The measured temporal profile and position of the laser pulse $(\mathrm{FWHM}=30 \mathrm{ps})$ is included at the bottom of the figure.

that required to avoid the presence of space charge effects.

\section{RESULTS}

In order to illustrate the capabilities of the developed experimental setup for the analysis of single event optical transients, we will show in this section a variety of results obtained during the study of rapid melting and solidification phenomena induced thin amorphous Ge films. The films were grown on glass and $\mathrm{Si}$ substrates in a dc magnetron sputtering system, as described in Ref. 29. Part of the results described in here have been discussed in previous works ${ }^{8,30,31}$ and thus we will concentrate on those aspects in which the advantages of the system can be most clearly appreciated.

Figure 4 shows a real-time reflectivity (RTR) transient recorded at $514 \mathrm{~nm}$ upon irradiation of a 50 -nm-thick amorphous Ge film with a single laser pulse at a fluence of 65 $\mathrm{mJ} / \mathrm{cm}^{2}$. We have also included in the same figure the temporal profile and position of the pump pulse ( $30 \mathrm{ps}$ FWHM) as measured in the same configuration in a highly scattering sample. The induced relative increase of reflectivity of $\approx 50 \%$ is completely consistent with the formation of an optically thick metallic molten layer at the surface. ${ }^{27}$ It is remarkable that the reflectivity rise, and thus the melting process, occurs within the pulse duration as predicted by several authors ${ }^{32,33}$ further confirming that the time resolution of the experiment is similar to the nominal one of the streak camera in this time window $(<7 \mathrm{ps})$. Similar results have been reported by time-resolved pump-and-probe measurements. ${ }^{10}$ Nevertheless, to our knowledge, this is the first time that a ps melting process has been observed in a real-time, single exposure experiment.

Apart from the capability of acquiring transient optical events in the ps time scale, the advantage of this experimental setup towards the pump-and-probe measurements or conventional real-time reflectivity measurements with fast photodiodes is also very clear when analyzing rapid solidification phenomena occurring in time a window of one to several tens of nanoseconds. Figure 5 shows the reflectivity evolution of a 50-nm-thick $a$-Ge film on a glass substrate under $30 \mathrm{ps}$ pulse at a fluence of $65 \mathrm{~mJ} / \mathrm{cm}^{2}$. Notice that in the case of a pump-and-probe experiment, the time window of the figure $(40 \mathrm{~ns})$ would require the scan of an optical delay line over a length $12 \mathrm{~m}$ with a separation of $9 \mathrm{~cm}$

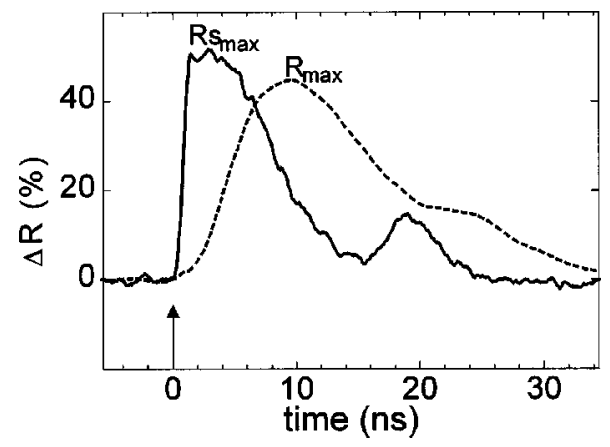

FIG. 5. Transient reflectivity changes upon melting and bulk solidification of an $a$-Ge film on glass substrate induced by a single ps laser pulse (65 $\mathrm{mJ} / \mathrm{cm}^{2}$ ) as measured simultaneously with sub-ns resolution (solid curve) and with ns resolution (dashed curve). The temporal position of the ps laser pulse is indicated by an arrow.

between consecutive data points for a time resolution of 300 ps. We have included in the figure for comparison both the evolution of the reflectivity as recorded with ns resolution (HeNe laser and photodiode) and with ps resolution $\left(\mathrm{Ar}^{+}\right.$ laser and streak camera) in the same time window. It is evident that neither the dynamics of the melting nor that of the solidification processes can be completely resolved with nsresolution measurements. The poor time resolution affects the observed reflectivity values at the maximum $R_{\max }$ which relate to the initial melt depth. ${ }^{13}$ Only when measured with sub-ns resolution (streak camera), it is clearly seen that melting occurs within the pulse duration and that the melt depth approaches the film thickness as indicated by the reflectivity increase of $R_{\max } \approx 50 \%{ }^{13,27}$ The decrease after the maximum of the RTR transients in Fig. 5 indicates the onset of solidification. While the ns-resolution transient shows the presence of a shoulder related to a recalescence process (release of latent heat) that slows down the solidification process, ${ }^{13,34}$ the sub-ns resolution transient instead reveals that the film solidifies (minimum in Fig. 5) before recalescence occurs (second maximum in Fig. 5). ${ }^{30}$ The surprising fact that massive recalescence occurs after the complete solidification of the film, instead of during solidification as suggested by other authors, ${ }^{35,36}$ is likely related to the combined use of ps laser pulses and a very thin film. Nevertheless, since this phenomenon can only be observed in the transients recorded with sub-ns resolution, it might have also been present in the studies of Refs. 35 and 36 although not being resolved.

Another example of the potential of the present experimental setup is provided by the results obtained in an $a-\mathrm{Ge}$ film with the same thickness but on a Si substrate. In this case, the film has been irradiated at an even higher fluence $\left(124 \mathrm{~mJ} / \mathrm{cm}^{2}\right)$ and Fig. 6 shows the corresponding RTR transients. The ns-resolution transient is quite similar to the one obtained in the film on glass substrate although the solidification process appears to be completed within a shorter time and no indication for recalescence can be seen. This behavior has earlier been related to the higher thermal conductivity of the Si substrate, ${ }^{37}$ which extracts both the laser induced heat and the solidification enthalpy much more efficiently than the glass substrate. The sub-ns resolution transient in Fig. 6 again reveals new features. The reflectivity decay after melt- 


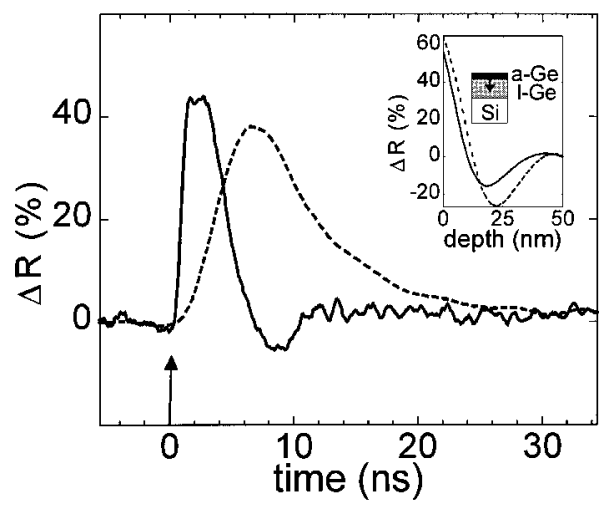

FIG. 6. Transient reflectivity changes upon melting and surface initiated solidification of an $a$-Ge film on Si substrate induced by a single ps laser pulse $\left(124 \mathrm{~mJ} / \mathrm{cm}^{2}\right)$ as measured simultaneously with sub-ns resolution (solid curve) and with ns resolution (dashed curve). The temporal position of the ps laser pulse is indicated by an arrow. The inset shows the optical simulations of the reflectivity evolution of the completely molten film at the corresponding wavelengths upon surface initiated amorphization propagating towards the substrate.

ing is followed by an oscillation below the initial level which suggests the presence of destructive interference effects of the probe beam. Similar oscillations have been observed upon explosive crystallization, ${ }^{38,39}$ during which a thin molten layer self-propagates into the amorphous material leaving crystalline material behind. Since the driving force for explosive crystallization is the amorphous-to-crystalline transition and in our case, the solidified material is amorphous, ${ }^{37}$ this scenario has to be excluded. Another possibility consistent with these oscillations is a solidification process initiated at the film surface and propagating in depth. ${ }^{40} \mathrm{We}$ have calculated $^{31}$ at both probe wavelengths the evolution of the reflectivity of the fully molten film upon surface initiated solidification with a solidification front propagating in depth. The result obtained (inset in Fig. 6) clearly shows that an oscillation has to be expected in both cases. The absence of such an oscillation in the transient recorded with ns resolution again confirms the need of sub-ns resolution measurements. In addition, the good agreement between the calculated reflectivity evolution and the one observed experimentally with sub-ns resolution strongly supports our interpretation of surface initiated solidification to take place. A similar solidification mechanism has already been observed to occur in 200 -nm-thick $a$-Si films. ${ }^{40}$ Nevertheless, the demonstration of surface initiated solidification in even much thinner films has only been possible due to the excellent time resolution of our experimental setup.

\section{ACKNOWLEDGMENTS}

This work has been partially supported by CICIYT and CSIC (Spain) under Project No. IN94-0205. One of the authors (J. S.) acknowledges with pleasure the funding of the European Union through a Marie Curie Fellowship (ERB40001GT54352) within the TMR Program.
${ }^{1}$ M. von Allmen and A. Blatter, Laser-Beam Interactions with Materials, 2nd ed., Springer Series in Materials Science Vol. 2, (Springer, Berlin, 1995).

${ }^{2}$ J. Shah, Ultrafast Spectroscopy of Semiconductors and Semiconductor Nanostructures, Springer Series in Solid State Sciences Vol. 115 (Springer, Berlin, 1996).

${ }^{3}$ See, for instance, Ultrashort Laser Pulses and Applications, edited by W. Kaiser, Topics in Applied Physics, Vol. 60 (Springer, Berlin, 1988) or any of the volumes of the series Ultrafast Phenomena like Ultrafast Phenomena XI, Springer Series on Chemical Physics Vol. 63 (Springer, Berlin, 1998).

${ }^{4}$ R. F. Wood and G. A. Geist, Phys. Rev. Lett. 57, 873 (1986).

${ }^{5}$ X. Liu, D. Du, and G. Mourou, IEEE J. Quantum Electron. 33, 1706 (1997).

${ }^{6}$ W. Kautek, J. Krüger, M. Lenzner, S. Sartania, Ch. Spielmann, and F. Krausz, Appl. Phys. Lett. 69, 3146 (1996).

${ }^{7}$ M. C. Morilla, C. N. Afonso, and J. Solis, Jpn. J. Appl. Phys., Part 2 36, L1015 (1997).

${ }^{8}$ J. Siegel, J. Solis, and C. N. Afonso, Appl. Phys. Lett. 75, 3102 (1999).

${ }^{9}$ E. N. Glezer, M. Milosavljevic, L. Huang, R. J. Finlay, T.-H. Her, and J. P. Callan, Opt. Lett. 21, 2023 (1996).

${ }^{10}$ C. V. Shank, R. Yen, and C. Hirlimann, Phys. Rev. Lett. 50, 900 (1983).

${ }^{11}$ J. Solis, M. C. Morilla, and C. N. Afonso, J. Appl. Phys. 84, 5543 (1998).

${ }^{12}$ M. C. Downer, R. L. Fork, and C. V. Shank, J. Opt. Soc. Am. B 4, 595 (1985).

${ }^{13}$ J. Siegel, J. Solis, and C. N. Afonso, J. Appl. Phys. 84, 5531 (1998).

${ }^{14}$ K. Sokolowski-Tinten, J. Solis, J. Bialkowski, J. Siegel, C. N. Afonso, and D. von der Linde, Phys. Rev. Lett. 81, 3679 (1998).

${ }^{15}$ K. Sokolwski-Tinten, J. Bialkowski, and D. von der Linde, Phys. Rev. B 51, 14186 (1995).

${ }^{16}$ E. K. Zavioski and S. D. Fanchenko, Sov. Phys. Dokl. 1, 285 (1956).

${ }^{17}$ J. P. Willson, W. Sibbett, and W. E. Sleat, Opt. Commun. 42, 208 (1982).

${ }^{18}$ Y. Kanemitsu, H. Kuroda, and S. Shionoya, Jpn. J. Appl. Phys., Part 1 23, 618 (1984).

${ }^{19}$ J. Solis, F. Vega, and C. N. Afonso, Appl. Phys. A: Mater. Sci. Process. 62, 197 (1996).

${ }^{20}$ D. J. Bradley, S. F. Bryant, J. R. Taylor, and W. Sibbett, Rev. Sci. Instrum. 49, 215 (1978).

${ }^{21}$ D. J. Bradley, S. F. Bryant, and W. Sibbet, Rev. Sci. Instrum. 51, 824 (1980).

${ }^{22}$ D. R. Hull and N. J. Freeman, J. Phys. E 13, 685 (1980).

${ }^{23}$ P. M. W. French, Rep. Prog. Phys. 58, 169 (1995).

${ }^{24}$ J. Siegel, Ph.D. thesis, Facultad de Ciencias Físicas de la Universidad Autónoma de Madrid/Instituto de Optica, CSIC, Spain, 1999.

${ }^{25}$ J. Solis, C. N. Afonso, S. C. W. Hyde, N. P. Barry, and P. M. W. French, Phys. Rev. Lett. 76, 2519 (1996).

${ }^{26}$ J. Solis and C. N. Afonso, J. Appl. Phys. 72, 2125 (1992).

${ }^{27}$ G. E. Jellison, Jr., D. H. Lowndes, D. N. Mashburn, and R. F. Wood, Phys. Rev. B 34, 2407 (1986).

${ }^{28}$ E. Huber and E. E. Marinero, Phys. Rev. B 36, 1595 (1987).

${ }^{29}$ J. C. G. De Sande, C. N. Afonso, J. L. Escudero, R. Serna, F. Catalina, and E. Bernabeu, Appl. Opt. 31, 6133 (1992).

${ }^{30}$ J. Siegel, J. Solis, and C. N. Afonso, Appl. Phys. Lett. 75, 3102 (1999).

${ }^{31}$ J. Siegel, J. Solis, and C. N. Afonso, Appl. Surf. Sci. (in press).

${ }^{32}$ D. Agassi, J. Appl. Phys. 55, 4376 (1984).

${ }^{33}$ N. Bloembergen, Mater. Res. Soc. Symp. Proc. 51, 3 (1986).

${ }^{34}$ T. Sameshima and S. Usui, J. Appl. Phys. 74, 6592 (1993).

${ }^{35}$ S. R. Stiffler, M. O. Thompson, and P. S. Peercy, Appl. Phys. Lett. 56, 1025 (1990).

${ }^{36}$ J. Boneberg and P. Leiderer, Phys. Status Solidi A 166, 643 (1998).

${ }^{37}$ J. Siegel, J. Solis, C. N. Afonso, and C. Garcia, J. Appl. Phys. 80, 6677 (1996).

${ }^{38}$ D. H. Lowndes, G. E. Jellison, Jr., S. J. Pennycook, S-P. Withrow, and D. N. Mashburn, Appl. Phys. Lett. 48, 1389 (1986).

${ }^{39}$ R. F. Wood and G. A. Geist, Phys. Rev. Lett. 57, 873 (1986).

${ }^{40}$ J. J. P. Bruines, R. P. M. van Hal, H. M. J. Boots, W. Sinke, and F. W. Saris, Appl. Phys. Lett. 48, 1252 (1986). 